\title{
生物柴油利用概况及其在中国的发展思路
}

\author{
贾虎森 许亦农 ${ }^{*}$ \\ (中国科学院植物研究所光合作用与环境分子生理学重点实验室,北京 10093)
}

\begin{abstract}
摘 要 石化燃料是当前人类使用的主要能源, 但其日益消耗殆尽, 同时造成了严重的温室效应和环境污染问题, 因此,生物柴油被当作石化燃料的绿色替代品, 许多国家都在大力研发。该文阐述了生物柴油的本质及其较石化 柴油咱使用上的优良特性, 综述了生物柴油主要在欧美国家中的发展现状及其它国家的研发动态, 特别是以大豆 (Glycine max) 和油菜 (Brassica campestris) 等油料作物为主的生物柴油原料生产状况。在分析了我国油料生产与食用 消费现状、受国际生物柴油大力发展的影响的程度及油料作物与粮食生产对耕地资源的激烈竞争矛盾的基础上, 提出了充分利用盐碱地、贫㾑、荒漠与退耕还林地,通过种植抗逆性强的油料植物发展我国生物柴油的思路。
\end{abstract}

关键词 能源 生物柴油

\section{WORLD BIODIESEL UTILIZATION AND DEVELOPMENT STRATEGIES IN CHINA}

JIA Hu-Sen and XU Yi-Nong *

( Key Laboratory of Photosynthesis and Environmental Molecular Physiology, Institute of Botany, Chinese Academy of Sciences, Beijing 100093, China)

\begin{abstract}
The stocks of fossil fuels, currently the major energy source driving the global socioeconomy, is being heavily depleted due to increasing anthropogenic activities worldwide, and its burning contributes to global climate warming and air pollution. In contrast, biodiesel is an alternative non-toxic, biodegradable, and renewable alternative energy source. This paper discusses the essence, properties and the development of biodiesel fuels, mainly in European countries; discusses the international supply and demand of soybean and rapeseed, the main biodiesel feedstock; and analyzes the production and consumption of edible plant oils in China and its balance as it is affected by international biodiesel development. Considering that domestic foodstuffs and plant oil production currently are insufficient to feed the 1.3 billion people in China due to limited agricultural lands, ultimately ways are needed to grow biodiesel feedstock on unproductive agricultural lands, such as saline-alkaline lands, poor soil, desertified soils, and plough-withdrawn land.
\end{abstract}

Key words Energy source, Biodiesel

能源是经济社会发展的重要动力, 面对全球社 会经济的迅速发展, 人类对能源的需求日益增长, 中 国能源消耗每年以超过 $10 \%$ 的速度增长 (静荷, 2004)。长期以来, 化石燃料一直是全人类消费的主 要能源。据世界 3 大石油公司埃克森-美孚、 BP 和 英荷壳牌 2003 年报预测, 按照目前已探明的世界石 油储量和开采速度, 全球石油的平稳供应只能维持 40.6 年 (生物质能, 2004)。另一方面, 化石燃料燃 烧时所产生的有害物质污染了环境, 导致温室效应、 全球气候变暖、生物物种多样性降低和荒漠化等诸 多生态问题, 严重影响着世界资源安全和社会经济 持续发展, 威胁着人类的生存。为满足社会发展对 能源的需求, 实现资源的永续利用, 维持和促进资
源、环境、社会经济的协调发展, 世界各国都在大力 研究和利用一种新型清洁能源一生物柴油。

生物柴油 (Biodiesel) 又称为阳光燃料 (Sunfuel), 据文献 (Bryant, 1976) 记载, 生物柴油最早在 1895 年 由德国工程师 Rudolf Diesel( $1858 \sim 1913$ ) 提出, 并在 1988 年由德国聂尔公司发明, 它是指油脂与醇类物 质 (通常为甲醇、乙醇) 经酯化反应 (Transesterification reaction)而获得的脂肪酸甲酯 (Quick, 1989) (图 1)。 生物柴油和石化燃料(煤炭天然气) 虽然都是由生物 有机质形成的, 从最源头上说都是光合作用的产物。 石化燃料是生物物质在地壳深层经过几亿年的变化 而最后形成, 生物物质成分复杂, 除碳、氢外, 还含 氮、硫、卤素和矿物质等; 生物柴油是用光合作用转 
化形成的油脂 (甘油三酯), 在人为条件下进行酯化 反应而加工得来。另外, 生物柴油本身含有大量氧 元素, 与石化柴油相比, 其组成中碳元素比例大大降 低。因此生物柴油燃烧耗氧量低, 燃烧充分, 温室气 体二氧化碳 $\left(\mathrm{CO}_{2}\right)$ 排放量小。而石化柴油燃烧时释 放大量 $\mathrm{CO}_{2}$, 以及硫氧化物 (Sulfur oxides, $\mathrm{SOx}$ )、一氧 化碳 (Carbon monoxide, CO)、微粒 (Particulate matter)、 氮氧化物 ( Nitrogen oxides, NOx)、多环芳烃(Polycyclic aromatic hydrocarbons, PAH) 和硝化多环芳烃 ( Nitrated polycyclic aromatic hydrocarbons, nPAH) 等有毒有害 物 $^{1)}$ (Faupel \& Kurki, 2002)。所以, 生物柴油具有使 用安全、清洁、高效的突出优点, 其发展可有效地保 护环境。

柴油分子由 15 个烃链组成, 植物油分子一般由 14 18 个烃链组成, 与柴油分子相似。生物柴油具 有燃料和润滑的双重功效、储存与运输安全、无须改 动柴油机而可直接添加使用等优点 (Kinast, 2003) (表 1)。<smiles>[R]C(=O)OCI(C)C(OC([R4])=O)OC([R4])=O</smiles>

Triglyceride

Methanol<smiles>[R]C(=O)OC</smiles>
(KOH)

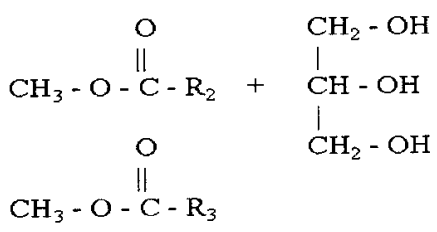

Mixture of fatty esters Glycerin

图 1 油脂酯化反应生产生物柴油

Fig. 1 Biodiesel produced by transesterification reaction

表 1 生物柴油和石化柴油的性能比较

Table 1 Comparison between biodiesel and petroleum diesel used as fuels

\begin{tabular}{|c|c|c|}
\hline 特性 Properties & 生物柴油 Biodiesel & 石化柴油 Diesel fuel \\
\hline 冷滤点 (CFPP)夏季产品 Cold filter plugging point (Summer) $\left({ }^{\circ} \mathrm{C}\right)$ & -10 & 0 \\
\hline 冷滤点 (CFPP) 冬季产品 Cold filter plugging point (Winter) $\left({ }^{\circ} \mathrm{C}\right)$ & -20 & -20 \\
\hline $20{ }^{\circ} \mathrm{C}$ 的密度 Density at $20^{\circ} \mathrm{C}\left(\mathrm{g} \cdot \mathrm{ml}^{-1}\right)$ & 0.88 & 0.83 \\
\hline $40{ }^{\circ} \mathrm{C}$ 动力粘度 Kinematic viscosity at $40{ }^{\circ} \mathrm{C}\left(\mathrm{mm}^{2} \cdot \mathrm{s}^{-1}\right)$ & $4 \sim 6$ & $2 \sim 4$ \\
\hline 闭口闪点 Flash point (closed cup) $\left({ }^{\circ} \mathrm{C}\right.$ ) & $>100$ & 60 \\
\hline 十六烷点 Cetane No. & $\geqslant 56$ & $\geqslant 49$ \\
\hline 热值 Calorific value $\left(\mathrm{MJ} \cdot \mathrm{L}^{-1}\right)$ & 32 & 35 \\
\hline 燃烧功效 (柴油 $=100 \%)$ Combustion efficacy! (diesel fuel =100\%) $(\%)$ & 104 & 100 \\
\hline 硫含量(质量分数) Sulfur content（Mass fsaction）(\%) & $<0.001$ & $<0.2$ \\
\hline 氧含量(体积分数) Oxygen content (Volume fraction) (\%) & 10 & 0 \\
\hline 燃烧 $1 \mathrm{~kg}$ 燃料按化学计算法的最小空气耗量 Minimal oxygen consumption of $1 \mathrm{~kg}$ fuel（kg） & 12.5 & 14.5 \\
\hline 水危害等级 Water hazard class & 1 & 2 \\
\hline
\end{tabular}

虽然生物柴油是用生物油脂生产的, 但从根本 上讲, 生物柴油的能量最初来源于太阳能, 绿色植物 通过光合作用来固定太阳能并为生产生物柴油提供 原料 (图 2), 它有效地解决了太阳能利用的两大难 点: 收集的高成本和间断性。据估计, 地球上每年植 物光合作用固定的能量达 $3 \times 10^{21} \mathrm{~J}$, 相当于世界能
源消费的 10 倍, 但植物固定的能量实际利用率还不 到 $1 \%$, 因此, 充分开发植物资源生产生物柴油潜在 巨大的发展空间。

\section{1 国外生物柴油的发展概况}

生物柴油是一种安全、清洁、高效的生物燃料,

1) Mittelbach M, Körbitz W (1995). Biodiesel: a regenerative fuel for unmodified diesel engines produced from a variety of fresh and used plant oils. Earth Conference on Biomass for Energy, Development and the Environment. Havana, Cuba. 


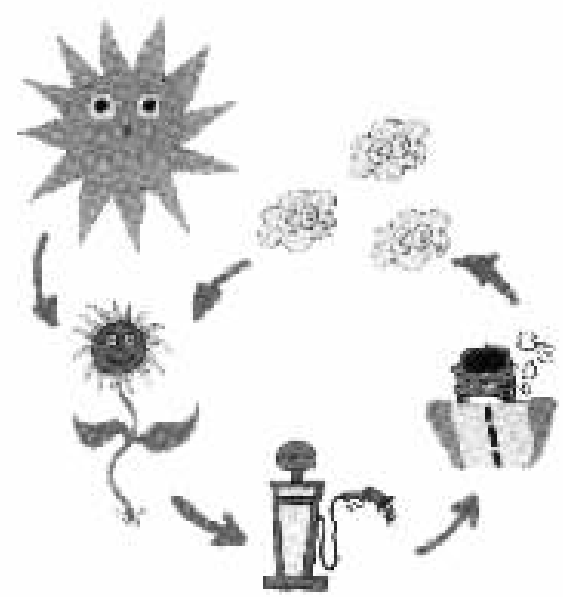

图 2 生物柴油的生产与再生 (Briggs \& Pearson, 2004)

Fig. 2 Produce and recycle of biodiesel (Briggs \& Pearson, 2004)

是典型的“绿色能源”, 因而成为石化燃料的替代品。 随着环境保护和石油资源枯竭两大难题越来越被关 注, 生物柴油已成为新能源研制和开发的热点, 近年 来发展极为迅速 (方芳等, 2004; 胡志远, 2004; 朱建 良和张冠杰, 2004)。

\section{1 北(南)美洲}

美国是最早研究应用生物柴油的国家, 1980 年 美国制定了国家能源政策, 明确提出以生物柴油替 代石化柴油战略, 目的在于促进本国可再生能源应 用。1990 年, 美国制定空气清洁法案, 要求降低柴 油燃料里硫含量和废气排放量, 从而促进生物柴油 投入商业应用(美国通过机动车排放立法控制尾气 污染, 2004)。据 2004 年美国国家生物柴油委员会 (National biodiesel board, NBB) 统计, 美国现有已投 产或正在建设中的商业化生物柴油厂 22 家, 另据美 国政府商品信用组织生物能源组 (US Government Commodity Credit Corporation (CCC) Bioenergy Program) 报告, 2003 年美国生产生物柴油 $6 \times 10^{7} \mathrm{~kg}$ (B100), 较 2002 年产量 $\left(3 \times 10^{7} \mathrm{~kg}\right)$ 增长了一倍。而美国能 源署计划, 到 2010 年将国内生物柴油产量提高到 $1.2 \times 10^{10} \mathrm{~kg}$ 。

巴西是最早掌握生物柴油技术的国家, 由 Ceará 大学 Expedito Parente 教授于 1980 年研究成功。随 后, 巴西政府推出“生物柴油计划”, 年产量曾一度达 到 $5 \times 10^{8} \mathrm{~kg}$, 但因生产成本过高, 市场需求不旺, 年
产减少到 $1 \times 10^{8} \mathrm{~kg}$ 。2003 年 7 月, 巴西政府重新启 动“生物柴油计划”, 巴西科技部计划生物柴油在 2020 年至少要占到市场份额的 $20 \%$, 目前一个日最 大生产 $5.6 \times 10^{6} \mathrm{~kg}$ 的生物柴油冶炼厂已经在北大 河州的 Mossoró 投入运营 (巴西政府重新启动生物柴 油计划, 2003)。

目前, 加拿大国内生物柴油开发技术已非常成 熟( Biodiesel Association of Canada, 2004)。Ocean Nutrition( 位于 Nova Scotia)、Milligan Bio-Tech(位于 Foam Lake, Saskatchewan)和 Rothsay Pilot(位于 Montreal)公 司都在生产生物柴油, 总产约 $1.5 \times 10^{7} \mathrm{~kg}$, 主要是自 产自用 ${ }^{1}$ 。Biox 公司采用 Toronto 大学开发的工艺, 于 2004 年在 Hamilton Ontario 开发建设年产 $6 \times 10^{7}$ $\mathrm{kg}$ 的生物柴油厂(赵卓然, 2003)。

南美洲的阿根廷也开始生产并应用生物柴油 （朱建良和张冠杰, 2004）。

\section{2 欧洲}

欧洲是目前世界上生物柴油生产及使用的主要 地区, 2003 年世界生物柴油总产量的一半以上就集 中在欧洲, 而这其中又以德国、法国、意大利和捷克 为主, 分别占到总生产量的 $50 \% 、 22 \% 、 18 \%$ 和 $5 \%$ (Biodiesel Association of Canada, 2004)。

德国对生物柴油研究应用十分突出, 1998 年, 德国的生物柴油产量还只有微不足道的 $5 \times 10^{7} \mathrm{~kg}$, 到 2003 年, 德国就已有 24 个生物柴油生产厂, 生产 $1.15 \times 10^{9} \mathrm{~kg}$, 成为世界最大的生物柴油生产国与出 口国 (Bockey, 2003)。全国有超过 1600 个生物柴油 加油站, 在萨尔州和下萨克州, 平均每 $20 \mathrm{~km}$ 就能找 到一个生物柴油加油站。近年来, 德国生物柴油产 量年均增长率都达到 40\% 50\%。德国政府的免 税政策(生产生物柴油企业享受全额免税), 使生物 柴油零售价低于石化柴油, 这极大地刺激并促进国 内生物柴油的生产应用(伊红, 2003)。

法国生物柴油生产曾处于世界领先地位, 但 2001 年以后被德国取代。法国生物柴油厂虽不多, 但生产能力很强。2003 年, 法国主要有 4 个生物柴 油厂, 生物柴油产量就达到 $3.1 \times 10^{8} \mathrm{~kg}$ 。法国在 Diester 兴建一个年生产 $1.6 \times 10^{7} \mathrm{~kg}$ 的生物柴油厂, 2005 年正式投产。2004 年 7 月, 法国制定国家生物 能源发展计划, 2007 年前, 1) 把国内用于生产生物 燃料的作物面积增加到 $1 \times 10^{6} \mathrm{hm}^{2}$, 从而超过德国,

1) Holbein BE, Stephen JD, Layzell DB (2004). Candian biodiesel initiative: aligning research needs and priorities with the emerging industry (Final Report). The BIOCAP Canada Foundation. 
成为欧洲最大的生物能源作物生产国; 2) 建设 4 个 年均生产 $2 \times 10^{8} \mathrm{~kg}$ 的新一代生物柴油厂, 力争 2010 年国内生物柴油产量达到 $8 \times 10^{9} \sim 10 \times 10^{9} \mathrm{~kg}$ ( 法国 欲成为欧洲最大的生物燃料生产国, 2004)。

意大利是欧洲第三大生物柴油生产国, 2003 年 国内有 9 个生物柴油生产厂, 年总生产能力为 3.3 $\times 10^{8} \mathrm{~kg}, 2003$ 年实际生产生物柴油 $2.73 \times 10^{8} \mathrm{~kg}$, 产 量比 2002 年增长了 $30 \%$ (我国生物柴油及产业化前 景分析,2005)。

捷克是目前欧盟 15 个新成员国中唯一生产生 物柴油的国家, 2003 年约生产生物柴油 $6 \times 10^{7} \mathrm{~kg}$ （我国生物柴油及产业化前景分析,2005)。

2002 年前, 西班牙已有两个生物柴油厂在商业 运营, 年生产能力分别为 $2 \times 10^{7} \mathrm{~kg}$ 和 $6 \times 10^{6} \mathrm{~kg}$ 。 2002 年 12 月 30 日政府颁布法令, 对生物燃料全部 免征特别税, 此举刺激了生物燃料行业的快速发展, 次年又有 4 个生物柴油厂开工兴建, 设计年生产能
力分别为 $1 \times 10^{8} \mathrm{~kg} 、 5 \times 10^{7} \mathrm{~kg} 、 3.5 \times 10^{7} \mathrm{~kg}$ 和 $5 \times 10^{6}$ $\mathrm{kg}$ 。2003 年, 西班牙生物柴油产量为 $1.8 \times 10^{7} \mathrm{~kg}$ 。 目前, 西班牙在东南沿海城市卡塔赫那的文斯贡布 雷拉斯山谷建成并投产一个年生产能力 $2.5 \times 10^{8} \mathrm{~kg}$ 的生物柴油厂( 我国生物柴油及产业化前景分析, 2005)。

英国 2003 年生产生物柴油 $3 \times 10^{3} \mathrm{~kg}$, 目前位于 苏格兰 Motherwell 的一个年产 $5 \times 10^{7} \mathrm{~kg}$ 的生物柴油 厂最近竣工生产, 另有 5 家生物柴油厂在拟建中 (庞 晓华 2004); 芬兰能源企业富腾公司 (Fortum) 正在南 部城市波尔沃兴建一座年 $1.7 \times 10^{8} \mathrm{~kg}$ 的生物柴油 厂, 预计 2007 年正式投产 (我国生物柴油及产业化 前景分析,2005)。

欧盟生物柴油委员会统计成员国中生物柴油的 主要生产情况 (表 2), 与 2003 年比较, 2004 年的生 产量大幅度提高 (Biodiesel Association of Canada, 2004)。

表 $22003 \sim 2004$ 年欧盟成员国的生物柴油生产情况

Table 22003 - 2004 biodiesel production in European Union

\begin{tabular}{lccc}
\hline 国家 Nation & $\begin{array}{c}2003 \text { 年产量 }\left(\times 10^{7} \mathrm{~kg}\right) \\
2003 \text { production }\left(\times 10^{7} \mathrm{~kg}\right)\end{array}$ & $\begin{array}{c}2004 \text { 年产量 }\left(\times 10^{7} \mathrm{~kg}\right) \\
2004 \text { production }\left(\times 10^{7} \mathrm{~kg}\right)\end{array}$ & $\begin{array}{c}\text { 增长率 }(\%) \\
\text { Increase rate }(\%)\end{array}$ \\
\hline 德国 Germany & 71.5 & 108.8 & 52.3 \\
法国 France & 35.7 & 50.2 & 40.6 \\
意大利 Italy & 27.3 & 41.9 & 53.5 \\
奥地利 Austria & 3.2 & 10 & 212.5 \\
西班牙 Spain & 0.6 & 7 & 1066.7 \\
丹麦 Danmark & 4.1 & 8.4 & 104.9 \\
英国 Great Britain & 0.9 & 1.5 & 66.7 \\
瑞典 Sweden & 0.1 & 0.8 & 700 \\
\hline
\end{tabular}

\section{3 亚洲}

在亚洲, 日本是发展生物柴油最早的国家, 也是 目前亚洲第一生物柴油生产大国。1999 年, 日本开 始了生物柴油的生产试验与商业开发。目前, 在日 本东京和长野有 4 家生物柴油工厂, 年生产生物柴 油 $4 \times 10^{8} \mathrm{~kg}$ (章文译, 2002)。

除日本外,亚洲许多国家正处于着手发展生物 柴油的起步阶段。印度政府在德国戴姆勒-克莱斯 勒公司的援助下, 正在实施“印度清洁空气计划”, 预 计 2005 年将进行商业化生物柴油生产; 泰国 UTICfoods 和 SKYfood 两食品公司计划与日本 PowwowPool 公司合作, 在泰国黎府进行商业化生产生物柴油, 而 泰国国营石油公司 PTT、泰国汽车技术者协会、泰国
海军部也正在联手开发生物柴油产业; 新西兰最大 国营电力公司一Meridian 能源电力公司通过新西 兰大学为其将开工的大型水电项目 (Project Aqua) 的 发电机和重型机械生产生物柴油, 新西兰 Barry Judd 公司也向政府提交了生物柴油发展计划; 韩国生产 的生物柴油已经在城市清洁车和垃圾运输车中试验 使用。另外, 菲律宾和印度尼西亚都在积极开发生 物柴油产业(朱建良和张冠杰, 2004)。

\section{4 非洲}

据报道, 总部设在英国伦敦的生物柴油公司 D1Oil 投资 300 万美元, 在南非东部港口城市德班修 建生物柴油厂, 计划 2005 年底建成投产, 这将是非 洲第一家生物柴油厂(李涁, 2004)。 


\section{2 生物柴油的主要原料}

生物柴油是生物油脂经甲酯化而形成的脂肪酸 甲酯, 所以任何形式的来自动物、植物和微生物的脂 肪酸都能用作生物柴油的原料。地球上, 动物和微 生物基本上都是直接或间接地以植物为食物的, 可 以说动物和微生物脂肪酸都是来源于植物。绿色植 物通过光合作用把太阳能直接转化为生物能, 从而 为其它生物 (动物和微生物) 提供生命的物质和能 量, 因此, 植物, 特别是油脂产量与含油量高的油料 植物, 才是生产生物柴油的最重要原料。目前, 生物 柴油主要是在美国和欧洲国家进行商业化生产与消 费,美国主要用大豆( Glycine max), 欧洲主要用油菜 (Brassica campestris), 大豆和油菜成为当前世界生产 生物柴油的主要原料。

\section{1 大豆}

大豆是一种重要的农作物, 种子含油量 16\% 18\%，而美国应用基因工程技术改良大豆使其含油 量提高到 20\% ( Su, 2000)。虽然含油量不是很高, 但 大豆种子中含有 $40 \%$ 左右蛋白, 加工生物柴油后的 豆粕是动物高蛋白饲料, 这使大豆种子能得到充分 利用,因而美国等许多国家都选用大豆作原料生产 生物柴油。据报道, 目前每 $1000 \mathrm{~kg}$ 大豆可生产得 到 $180 \mathrm{~kg}$ 豆油和 $880 \mathrm{~kg}$ 豆粕 $(\mathrm{Su}, 2000)^{1)}$ 。

美国是世界大豆生产第一大国,近 5 年平均年 产量约 $7.8 \times 10^{10} \mathrm{~kg}$ (陈智文 2005), 国内 $55 \%$ 以上 的生物柴油是用大豆生产的 $(\mathrm{Su}, 2000)^{1)}$ 。根据 Sheehan 等 (1998) 研究, 在良好生产条件下, 用精炼 生物油脂生产出优质生物柴油的比率可以高达 99.9\%。粗略地把生物油脂的生物柴油产出率用 $100 \%$ 来考虑, 结合大豆平均 18\% 含油量, 根据美国 国家生物柴油委员会 (National Biodiesel Board, NBB) 公布的数据, 2003 年生物柴油 (B100) 产量 $8 \times 10^{7} \mathrm{~kg}$ (即 $4 \times 10^{8} \mathrm{~kg}$ B20 生物柴油), 计算可知美国 2003 年 生产生物柴油实际消耗大豆 $8 \times 55 \% \div 18 \% \div 100 \%$ $=2.4 \times 10^{8} \mathrm{~kg}$, 约占该国大豆总产量 $\left(7.8 \times 10^{10} \mathrm{~kg}\right)$ 的 $0.3 \%$ 。目前, 美国大豆有很大部分直接以未加 工产品直接出口, 每年约 $2.4 \times 10^{10} \mathrm{~kg} \sim 2.8 \times 10^{10}$ $\mathrm{kg}$, 占总产量 $\left(7.8 \times 10^{10} \mathrm{~kg}\right)$ 的 $1 / 3$ 左右。

巴西和阿根廷是世界第二和第三大豆生产大
国, 年产大豆约 $4.2 \times 10^{10} \mathrm{~kg}$ 和 $3.0 \times 10^{10} \mathrm{~kg}$ 。目前, 这两国生物柴油基本处于起步发展时期, 因此还未 见两国大豆使用在生物柴油上的报道。在这两个国 家生产的大豆中, 出口占去了很大的比例, 2003 年 巴西出口大豆、豆油和豆粕分别为 $2.1 \times 10^{10} \mathrm{~kg} 、 1.4$ $\times 10^{10} \mathrm{~kg}$ 和 $2.4 \times 10^{9} \mathrm{~kg}$, 阿根廷也分别出口了 $7 \times$ $10^{9} \mathrm{~kg} 、 4.17 \times 10^{9} \mathrm{~kg}$ 和 $1.95 \times 10^{10} \mathrm{~kg}$ (朱建良和张冠 杰, 2004)。

\section{2 油菜}

生物柴油最先是由德国聂尔公司在 1988 年用 菜䊏油生产诞生的 ${ }^{2}$, 目前油菜仍在国际生物柴油 生产中占统治地位, 这归因于欧洲是世界生物柴油 的主要生产地, 而油菜又是欧洲生物柴油的主要原 料。当前欧洲地区生物柴油 $80 \%$ 以上是用油菜生 产的, 而法国和德国生产的油菜䊏分别就有 $27 \%$ 和 40\%用于生产生物柴油。

在世界油料作物中, 无论是面积, 还是总产量, 油菜都仅次于大豆,位居第二。据报道,一些优良油 菜品种其种子含油量可超过 50\%（Wolfgang \& Wilfried, 1998), 但总体来说, 世界油菜籽平均含油量在 $40 \%$ 左右, 另含约 $40 \%$ 蛋白。1 $000 \mathrm{~kg}$ 油菜籽除了 可生产出 $400 \mathrm{~kg}$ 菜籽油外, 还可得到 $600 \mathrm{~kg}$ 菜籽粕 用作饲料 (Kinast, 2003)。

中国油菜种植面积和总产量均位居世界第一, 但国内油菜单产仍低于世界平均水平, 品种含油量 偏低 (陈锦清和黄锐之, 2004)。目前, 中国生产油菜 不能满足国内食用需求, 据海关统计数据, 2003 年 中国进口油菜和菜油均达到 $2.5 \times 10^{9} \mathrm{~kg}$ 左右。

\section{3 其它生物柴油原料}

由于生物柴油是生物油脂的酯化物, 这就决定 了任何来源的生物油脂都有可能作为生物柴油原 料。不同国家根据自己的生物资源条件，已经开辟 了多种生物原料来发展生物柴油。

动物脂肪: 位于加拿大 Nova Scotia 的 Ocean Nutrition Operation 公司利用海洋鱼类脂肪在自产自用 生物柴油,而畜牧业发达的澳大利亚和新西兰利用 牛羊肉食加工中产生的动物脂肪副料进行生物柴油 生产(Biodiesel Association of Canada, 2004)。

其它植物油脂: 除了大豆和油菜外, 许多国家计 划或已经在用其它特种植物生产生物柴油, 如棕榈 
(Trachycarpus fortunei)(印尼、马来西亚)、椰子(Cocos nucifera)(菲律宾)(刘宗, 2004)、苞麻( Ricinus commu$n i s$ )(巴西)、麻疯树 (Jatropha curcas)(泰国)、向日葵 (Helianthus annus) (巴西)、棉籽 (Gossypium arboreum) (印度)(董伟,2004)。

工程微藻: 美国可再生能源国家实验室(NREL) 通过现代生物技术建成“工程微藻”, 即硅藻类的一 种“工程小环藻”, 在实验室条件下可使脂质含量达 $60 \%$ 以上, 户外生产也可达 40\%以上。预计每英亩 “工程微藻”每年可生产 $5 \times 10^{3} \mathrm{~kg} \sim 13 \times 10^{3} \mathrm{~kg}$ 生 物柴油 (宋玉春, 2004)。

废食用油(地沟油): 日本在东京和长野建成 4 家生物柴油工厂, 目前利用饭店废食用油(地沟油) 年生产生物柴油 $4 \times 10^{8} \mathrm{~kg}$ (章文译, 2002)。

\section{3 中国发展生物柴油的思路}

\section{1 中国发展生物柴油的迫切性}

据 2005 年 5 月 “北京第八届科博会中国能源战 略高层论坛” 会议, 亚太地区将成为世界石油供需 矛盾最尖锐地区, 该区目前石油剩余探明储量只占 世界的 $3.7 \%$, 而石油产量占世界的 $10.7 \%$, 消费量 占世界的 $28.1 \%$, 自给率仅为 $38.1 \%$ 。近年来, 我 国石油消费逐年增长, 2004 年石油表观消费量达 $2.92 \times 10^{11} \mathrm{~kg}$, 同比增长 $15.5 \%$; 而原油产量仅 1.75 $\times 10^{11} \mathrm{~kg}$, 同比增长 $2.9 \%$; 进口石油 $1.23 \times 10^{11} \mathrm{~kg}$, 同比增长 $34.8 \%$, 原油对外依存度已达 $42.1 \%$ 。根 据国家发展和改革委员会能源研究所预测, 2020 年 中国石油的需求量将为 $4.5 \times 10^{11} \mathrm{~kg} \sim 6.1 \times 10^{11} \mathrm{~kg}$, 届时国内石油产量估计为 $1.8 \times 10^{11} \mathrm{~kg}$, 进口量将为 $2.7 \times 10^{11} \mathrm{~kg} \sim 4.3 \times 10^{11} \mathrm{~kg}$, 进口依存度将处于 $60 \%$ 70\%(潘家华, 2004)。

目前全球石油主要消耗在运输业, 美国运输业 的石油消费比例接近 $70 \%$, 我国目前交通能源消费 占柴油产量的 $63 \%$ 以上,汽油产量的 $92 \%$ 以上。中 国经济的迅猛发展,已经从“自行车王国”逐渐走上 了“汽车大国”。据预测, 中国的汽车保有量以每年 超过 $10 \%$ 的速度增长, 2005 年可能达到 $2.5 \times 10^{7}$ 辆。还有人做了一个简单的预测, 如果 2020 年全国 达到小康水平, 保守地估计中国人均汽车保有量应 该有可能达到目前世界的人均水平, 约每千人 100 辆, 中国的汽车保有量将是 $1.4 \times 10^{8}$ 辆, 比现在增 长 6 倍(张小明, 2004)。

石油产品在机动车辆中的大量使用, 是造成温 室效应和环境污染的主要元素(石克轩, 2004)。根
据我国环保部门提供的数据, 在北京、上海和广州等 大城市, 机动车对 $\mathrm{CO} 、 \mathrm{HC} 、 \mathrm{NO}_{\mathrm{X}}$ 的贡献率已经超过 $50 \%$, 有的已达到 $90 \%$ 以上。鉴于机动车使用石油 对环境造成的严重污染, 柴油车已经成为今后汽车 发展的主流(张小明, 2004)。提起柴油车, 它并不是 许多人脑海中冒着黑烟、噪声巨大、肮脏不堪的环境 污染罪鬼 (葛蕴珊等, 2004)。法国标致-雪铁龙集 团、美国德尔福集团、美国霍尼韦尔涡轮增压系统有 限公司、美国康明斯公司、德国博世汽车部件有限公 司等世界顶尖的汽车厂商和发动机制造商的总裁、 经理、工程师, 以及国内天津大学内燃机国家重点实 验室、清华大学汽车安全与节能国家重点实验室的 专家、学者都明确指出, 柴油发动机具有比汽油发动 机更好的燃油经济性、安全性, 更环保、更清洁。柴 油机能源消耗为汽油机的 $45 \% \sim 60 \%$, 且二氧化碳 排放量比汽油机大约低 30\% 35\%, 环境效益十分 显著, 这是目前柴油汽车发展迅速的主要原因(王 威,2004)。如果北京的 $6 \times 10^{4}$ 多辆出租车全部更 换为满足欧 Ш标准的柴油出租车, 那么北京每年将 可减少燃油耗 $1.13 \times 10^{8} \mathrm{~kg}$, 每个司机可节省运营燃 油费用 5600 元, 减排二氧化碳 $3.4 \times 10^{7} \mathrm{~kg}$ 。根据 欧盟的统计, 欧盟主要国家的柴油轿车市场份额从 1991 年的 $16 \%$ 快速增长到 2003 年的 $44 \%$, 柴油汽 车已占商用车的 $90 \%$ 、乘用车的 $32 \%$ (王威, 2004)。

与汽油车相比, 柴油汽车的优越性不只限于当 前水平的高效率、低油耗和低污染, 基于生物柴油较 石油的优良特性, 今后生物柴油的使用, 必将柴油汽 车的优越性推向更高层次。目前, 生物柴油的使用 主要受价格限制(宋玉春, 2004), 但是发展柴油汽车 和使用生物柴油将是必然。在 2004 必比登挑战赛 中, AudiA21.2TDI、途锐、AudiA83.0TDIQuattro, 高尔 夫 IV、Xsara 毕加索等世界汽车巨头的名牌车辆都用 生物柴油作然料 (2004 必比登挑战赛: 新能源汽车 大赛上海开战, 2004)。

在柴油轿车方面, 中国目前还是空白, 潜在巨大 发展空间, 戴姆勒- 克莱斯勒、菲亚特、福特、德国大 众、本田、通用汽车、标致雪铁龙、宝时捷、雷诺、丰 田、尼桑、马自达等全球著名汽车公司在争先开拓和 占据中国柴油汽车市场 (李绍纯, 2003)。

\section{2 中国发展生物柴油的关键问题}

生物柴油经过多年的研究和发展, 其生产技术 和使用技术已比较成熟 (王一平等, 2003)。早期的 高温裂解法最为简单, 普通家庭就可以进行小作坊 生产(Kinast, 2003)。现在生物柴油生产技术主要可 
以归结为两个方面: 物理法和化学法。物理法是利 用了生物油脂高能量密度和可燃烧的特性, 不改变 生物油脂的组成和性质, 直接把生物油脂与石化柴 油混合使用, 或把生物油脂制成微乳液而作为燃料 使用 (Zaffroni et al ., 1987)。化学法是把生物油脂和 甲醇等低碳一元醇 (通常为 C1-4 醇) 进行酯化或转 酯化反应,生成相应的脂肪酸低碳烷基酯,再经分离 甘油、水洗、干燥等适当处理后而获得生物柴油。生 物柴油化学生产技术经过多年发展, 已经形成比较 完备的技术体系和方法,涵盖了化学催化剂法、生物 酶催化剂法、无催化剂法(在高温高压下进行)、常压 法和加压法等多个方面。化学法中的常压连续转酯 化和加压连续转酯化生产技术, 已在欧美等发达国 家形成大规模工业化生产,代表了当今主流生物柴 油技术,而且技术仍在不断发展。

我国生物柴油生产技术并不落后于欧美等发达 国家,例如, 脂肪酸烷基酯的生产方法、棉籽油皇脚 料合成脂肪酸甲酯的专利技术、高酸值动植物油脂 共沸蒸馏酯化-甲酯化技术、短链脂肪酸酯作为酰基 受体的酶法生物柴油技术等(孟凡清等, 2003; 雷猛 等,2004; 宋玉春, 2004)。清华大学化工系再生资源 与生物能源试验室的一条全新生产工艺路线的生化 酶催化剂生产生物柴油工艺问世, 并在湖南海纳百 川生物工程有限公司生物柴油中试装置上得到成功 应用( 孙可华,2004)。

可见，技术与设备条件不是限制我国发展生物 柴油的主要问题,而真正的限制因素是原料,如何获 得充足量的生物油脂才是我国生物柴油产业的瓶 颈。

世界生物柴油发达国家都是以植物油为主要生 产原料。我国是一个以种植业为支柱的发展中农业 国家，全国畜牧和养殖业的比例很小，所以动物油脂 不可能成为我国发展生物柴油的主要原料。另外, 我国植物油脂生产不足（郭洪涁，2004）。中国虽然 是全球最大的油菜籽、棉籽以及花生生产国, 以及全 球第四大豆生产大国, 但油料生产不能满足人们食 用需求。近几年来,生活水平的提高, 我国人均植物 油消耗量增长较快, 2002 年达到 $15.9 \mathrm{~kg}$, 但远低于 亚洲其它国家和地区, 如香港 (40.8 $\mathrm{kg}$ ) 和台湾 (34.9 $\mathrm{kg}$ )(王俊籽, 2004)。据统计 2004 年我国人均植物 油消耗量 $17.6 \mathrm{~kg}$, 则 13 亿人口食用植物油总需求 为 $2.3 \times 10^{10} \mathrm{~kg}$, 而国内油脂生产量仅 $1.4 \times 10^{10} \mathrm{~kg}$ 。 为此, 2004 年全年进口食用油脂 $6.76 \times 10^{9} \mathrm{~kg}$, 其中 进口豆油 $2.52 \times 10^{9} \mathrm{~kg}$, 菜籽油 $3.5 \times 10^{8} \mathrm{~kg}$, 棕榈油
进口 $2.4 \times 10^{9} \mathrm{~kg}$, 进口大豆 $2 \times 10^{10} \mathrm{~kg}$, 油菜籽 $4.7 \times$ $10^{8} \mathrm{~kg}$ (浅析我国豆油进口与大豆进口的相关性, 2005）。油料生产能力不足将是限制我国生物柴油 发展的主要因素。

油料是人们食用必需品, 在农产品中价格地位 本身就较高, 这抬高了其加工的生物柴油价格, 其中 $75 \%$ 就是成本, 即油料本身的价格 (宋玉春, 2004)。 在国外生物柴油发达的国家, 政府对生物柴油产业 给予免税或其它优惠政策。因此, 我国要发展生物 柴油, 除了解决原料这一根本问题外, 还需要政府对 该产业进行引导与扶持。

3.3 油料作物成为中国生物柴油主要原料的可能 性

要发展生物柴油, 中国到底能否通过增加大豆 和油菜等作物的生产而提供足够的植物油呢? 以下 情况在一定程度上已经否定了这种可能。

1)目前中国植物油脂的食用缺口太大。尽管当 前中国人均食用油消费水平较低, 但国内生产仍不 能满足消费需要。2004 年食用植物油缺口为 $8.88 \times$ $10^{9} \mathrm{~kg}$, 高达国内总生产能力的 $63.4 \%$ 。这决定了今 后很长时间中, 中国食用植物油生产只能是力争满 足人们生活需要, 不可能出现足量剩余植物油用来 发展生物柴油。

2)中国植物油脂的增产潜力不足。不否认我国 目前种植大豆和油菜的平均含油量及单产较世界平 均水平都低, 这使我国植物油的增产具有一定潜力, 但其开发仍然有限, 即使完全达到国际较高的油籽 含油量和单产水平, 以此估算, 这将仍然难以弥补目 前巨大的食用消费缺口。通过扩大油料作物种植面 积发展生物柴油难以实现。2004 年度全国土地利 用变更调查结果显示, 中国大陆耕地面积又减少 2.5 $\times 10^{4} \mathrm{~km}^{2}$, 仅有耕地 $1.23 \times 10^{6} \mathrm{~km}^{2}$, 约占世界耕地面 积的 $5 \%$, 耕地面积已经降至专家们公认的 $1.20 \times$ $10^{6} \mathrm{~km}^{2}$ 警戒线。而我国有 13 亿人口, 是世界人口 总量的 $21 \%$ 。以全世界 $5 \%$ 的耕地养活 $21 \%$ 的人 口, 这已经对我国土地生产造成很大压力。我国谷 物类粮食由净出口转变为净进口(表 3)(中国 2004 年粮食进口,2005）。“粮食安全”永远是关系国家经 济发展和社会长治久安的大事, 因而, 油料作物与粮 食生产存在严重的耕地竞争的情况下, 扩大油料种 植面积的可能性就很小了。

\section{4 中国获取生物柴油原料的思路}

中国要发展生物柴油不可能向耕地要原料,而 
表 $32003 \sim 2004$ 中国粮食净进口变化 $\left(\times 10^{7} \mathrm{~kg}\right)$

Table 3 Change of China net foodstuff importation in 2003 and $2004\left(\times 10^{7} \mathrm{~kg}\right)$

\begin{tabular}{ccc}
\hline 粮食净进口量 & \multicolumn{2}{c}{ 年 Year } \\
\cline { 2 - 3 } Net foodstuff importation & 2003 & 2004 \\
\hline 合计 Total & -44 & 2412 \\
谷物 Corns & -1987 & 502 \\
小麦 Wheat & 45 & 726 \\
稻谷 Rica & -236 & -14 \\
玉米 Maize & -1640 & -232 \\
大麦 Barley & 136 & 171 \\
豆类 Beans & 1943 & 1910 \\
大豆 Soybean & 2047 & 1990 \\
杂豆 Other beans & -104 & -80 \\
\hline
\end{tabular}

植物的生长却又离不开土壤, 因此, 要获得发展生物 柴油的植物油脂原料就只能向非耕土地索取。

4.1 利用荒漠化 (盐碱化) 土地, 发展中国生物柴油 原料

中国目前存在荒漠化 (盐碱化) 土地 $2.6 \times 10^{6}$ $\mathrm{km}^{2}$, 占国土总面积的 $27.32 \%$, 并以每年 $3.0 \times 10^{3}$ $\mathrm{km}^{2}$ 的速度在扩大 (世界气象日: 我们的目光投向可 再生能源, 2005)。据 2004 年中国国土资源公报, 全 国现有耕地 $1.224 \times 10^{6} \mathrm{~km}^{2}$ (其中约 $10 \%$ 为荒漠化 耕地), 荒漠化土地面积是全国耕地面积 (1.224 × $\left.10^{6} \mathrm{~km}^{2}\right)$ 的 2 倍多。有限的耕地面积, 注定广阔的荒 漠化 (盐碱化)土地必将成为中国发展生物柴油原料 的主要载体。在中国境内, 分布着多种特别抗盐碱、 抗干旱的野生油料植物, 如盐角草 ( 又名海蓬子, Salicornia), 耐盐度高达 $6.5 \%$, 种子含油量约 $30 \%$; 油莎豆 ( 又名油沙草, Cyperus esculentus) 耐旱涝、㾑 瘦、盐碱, 其核状根茎果油量约 20\% 30\%。总之, 中国有许多抗逆性强的野生油料植物, 通过政府引 导, 严格的科学规划, 相信一定能在广阔的荒漠化 (盐碱化) 土地上将生物柴油原料蓬勃发展起来。

\section{2 在退耕还林中种植木本油料植物来发展生物} 柴油原料

中国的山地丘陵多, 山地资源丰富, 山地、高原 和丘陵约占国土总面积的 $69 \%$ 。由于山地土层薄、 肥力差和缺水等因素一定程度地限制了植物生长, 加之过去对林木的乱砍乱伐, 致使许多地方植被退 化, 水土流失。据统计, 目前我国森林覆盖率只有世 界平均水平的 $62 \%$, 人均森林面积只有世界平均水 平的 $20 \%$, 国内水土流失面积已占到国土面积的 $38 \%$ 。面对日益恶化的生态环境, 我国当前正在大 力实施退耕还林工程, 2004 年我国退耕 $7.3 \times 10^{3}$ $\mathrm{km}^{2}$, 占总耕地减少量 $\left(9.5 \times 10^{3} \mathrm{~km}^{2}\right)$ 的 $77.3 \%$ 。结 合对发展生物柴油的迫切需要及有限耕地资源对发 展生物柴油原料的限制, 在退耕还林中种植木本油 料植物是中国发展生物柴油原料的重要出路, 也是 有效解决“三农”问题的蹊径。退耕地虽然贫痊, 作 物生长极大受限, 而我国一些野生木本油料植物具 有很强抗逆性, 耐旱、耐贫痊, 如黄连木 (又名楷树, Pistacia chinensis), 耐旱、耐痊薄, 果壳、种子和种仁 含油量分别为 $3 \%$ 、35\% 和 $56 \%$; 文冠果 ( 又名崖木 瓜, Xanthoceras sorbifolia), 抗寒、抗旱和抗盐碱, 种子 含油率高达 $50 \%$; 麻疯树 ( 又名小桐籽, Jatropha curcas), 抗旱, 种子含油量高达 52.4\% (董伟, 2004)。 在退耕还林中, 依据科学分析, 因地制宜, 合理区划 发展特种抗逆油料植物, 将是我国获取生物柴油原 料的主要出路。

从全球能源形势与发展趋势来看, 生物柴油是 大力开发的一种重要绿色能源。原料是我国发展生 物柴油需要解决的主要问题。我国人口多, 耕地少, 国内不只是油料作物生产不能满足人们的食用消 费, 而且粮食也不能完全自给自足。扩大油料作物 生产势必造成与粮食对有限耕地的竞争, 这种状况 决定了我国不具备象欧美等国家那样专门种植大 豆、油菜等油料作物来发展生物柴油的条件。为维 持和促进资源、环境、社会经济的协调发展, 充分开 发和利用大面积的荒漠与盐碱化土地, 以及退耕还 林地, 针对性地选种特种油料植物可能是我国获取 大量生物柴油原料的重要思路。

\section{参 考 文 献}

Analysis on the relationship between soybean oil and soybean importation(浅析我国豆油进口与大豆进口的相关性) (2005). http: //www. oilcn. com/detail. aspx? Id = 35331. Cited 9 May.

Biodiesel Association of Canada (2004). Economic, financial, social analysis and public policies for biodiesel. http://www. biodiesel-canada. org/resources/publication-files/20041001-nrcanbiodieselphase1.pdf. Cited Nov.

Biodiesel in China and its prospect(我国生物柴油及产业化前景 分析) (2005). http://www. newenergy. org. cn/energy1/20055/20055659.html. Cited 8 May.

Biomass (生物质能) (2004). http://database. cpst. net. cn/popul/views/artic/41225161430. html. Cited 25 Dec.

Bockey D (2003). Situation and development potential for the production of biodiesel - an international study. http: //www. ufop. de/download/FAL-Bockey-English. pdf.

Briggs MS, Pearson J (2004). Incorporation lessons on biodiesel into the science classroom. The NH Science Teacher Association (NHSTA) Annual Conference, Philips Exeter Academy, Exeter. 
Bryant L(1976) . The development of the diesel engine. Technology and Culture, 17, $432-446$.

Challenge Bibendum 2004: Rallying together towards sustainable mobility in Shanghai (2004 必比登挑战赛: 新能源汽车大赛 上海开战）（2004）. http://auto. tom. com/2150/2166/ 20041110-62479. html. Cited 10 Nov.

Chen JQ(陈锦清), Huang RZ(黄锐之) (2004). Genetic engineering on oil crop breeding. China Biotechnology (中国生物工程 杂志),24(5),24-29. (in Chinese with English abstract)

Cheng ZW (陈智文) (2005). Status quo of production and trade of soybean in America. World Agriculture (世界农业) , 308(1), 15 - 17. (in Chinese)

China net foodstuff importation in 2004 (中国 2004 年粮食进口) ( 2005 ). http://www. sannong. gov. cn/qufb/nysc/ 200506020039. htm. Cited 6 Apr.

Dong W(董伟) (2004). Biodiesel can be obtained from Jatropha curcas L. Energy Research and Information (能源研究与信息), 20(2),92. (in Chinese)

Fang F(方芳), Yu J (余剑), Zeng HY (曾虹燕) (2004). Study and application of biodiesel. Environmental Protection in Transportation (交通环保),25(5),45 - 47. (in Chinese with English abstract)

Faupel K, Kurki A (2002). Biodiesel: a brief overview. http:// www . agroindonesia. com/Publications/biodiesel. pdf.

France plans to be the biggest biofuel production country in Europe. (法国欲成为欧洲最大的生物燃料生产国) (2004). http:// www. people. com. cn/GB/keji/1059/2772225. html. Cited 9 Sept.

Ge YS(葛蕴珊), Zhang SY (张世鹰), Hao LJ(郝利君), Wu SJ (吴思进), Kong LH(孔林河) (2004). Application investigation of biodiesel on a diesel engine. Neiranji Gongcheng (内燃机工 程),25(2),12-14. (in Chinese with English abstract)

Guo HB(郭洪涁) (2004). Analysis of China vegetable fats and oil crops importation and exportation in 2003. China Oils and Fats (中国油脂),29(4),68-69. (in Chinese)

Hu ZY (胡志远) (2004). Utilization and development of biodiesel fueled by cars. Automobile Research \& Development (汽车研究 与开发), (11),27-31. (in Chinese)

Jing H(静荷) (2004). Lack of energy cannot be dispelled in short time and price of petroleum will still maintain at a high level. China Petroleum and Chemical Industry Economic Analysis (中国 石油和化工经济分析),(19),56。(in Chinese)

Kinast JA(2003) . Production of biodiesels from multiple feedstocks and properties of biodiesels and biodiesel/diesel blends. http:// www. nrel. gov/docs/fy03osti/31460. pdf.

Lei M(雷猛), Xu SH (许世海), Wei XP(魏小平) (2004). Biodisel produced by vegetable seeds oil and its properties. Journal of Logistical Engineering University (后勤工程学院学报), 18(4) ,59-63. (in Chinese with English abstract)

Li B(李涁) (2004). South Africa: soy used as diesel. Ecological Economy (生态经济),,(5),17(in Chinese)
Li SC(李绍纯) (2003). Market foreground of diesel cars in China. China Auto Market (中国汽车市场) (5),19. (in Chinese)

Liu Z(刘宗) (2004). Philippine and United States jointly develop biodiesel using coconut as foodstock. World Agriculture (世界农 业) ,307(11),61-62. (in Chinese)

Meng FQ (孟凡清), Wang DM (王德民), Zhang DN (张大年) (2003) . Study progress on biodiesel in China. Shanghai Chemical Industry (上海化工), (12),30 - 33. (in Chinese with English abstract)

National Biodiesel Board (NBB) (2004). DaimlerChrysler set energy security example. http://www. biodiesel. org/resources/ pressreleases/pas/20041117-jeep-liberty-ride-drive. pdf. Cited 17 Nov.

Pan JH(潘家华) (2004). Energy problems in China and national petroleum reserve. Oil \& Gas Storage and Transportation(油气 储运),23(12), 1-3. (in Chinese)

Pang XH (庞晓华) (2004). UK biofuels factory plans to build 5 biodiesel factories. Environmental Protection of Chemical Industry (化工环保) ,24,339. (in Chinese)

Pollution protected by emission control regulations in United States. (美国通过机动车排放立法控制尾气污染) (2004). http:// news. sina. com. cn/c/2005-04-14/10085647506s. shtml. Cited 14 Apr.

Project of biodiesel rerun in Brazil(巴西政府重新启动生物柴油 计划 ) (2003). http://br. mofcom. gov. cn/aarticle/ztdy/ 200307/20030700113699. html. Cited 31 Jul .

Quick GR(1989). "Oilseeds as Energy Crops" in Oil Crops of the World. McGraw-Hill Publishing Co. New York 118-131.

Sheehan J, Camobreco V, Duffield J, Graboski M, Shapouri H (1998). An overviem of biodiesel and petroleum diesel life cycles. A joint study sponsored by US Department of Agriculture and US Department of Energy. http: //www. nrel. gov/docs/ legosti/fy98/24772. pdf. Cited 20 May.

Shi KX (石克轩) (2004). Alternative fuels for cars. Sinopec (中 国石化), 228(9),46-49. (in Chinese)

Song YC(宋玉春) (2004). Biodiesel in China and its foreground analysis. China Petroleum and Chemical Industry Economy Analysis (中国石油和化工经济分析),(16),16-19. (in Chinese)

Su Y (2000). Supply and demand of soybeans as feedstock for soy diesel. http://www. mda. state. mn. us/ams/soydieselreport. pdf.

Sun KH(孙可华) (2004). Tsinghua University a new bio-eneyze catalyzed biodiesel production technics is passed. Guoneiwai Shiyou Huagong Kuaibao (国内外石油化工快报), 34(8), 2728. (in Chinese)

Wang JZ(王俊籽) (2004). Analysis on edible plant oil supply and demand. Machinery for Cereals Oil and Food Processing (粮油加 工与食品机械), (3),12-15. (in Chinese)

Wang W(王威) (2004). Development of biomass energy is urgently needed. New Economy Weekly (新经济导刊), (21), 44 - 46. (in Chinese) 
Wang YP(王一平), Zhai Y(翟怡), Zhang JL(张金利), Li W(李 伟), Han ZT(韩振亭) (2003). Research progress of biodiesel preparation. Chemical Industry and Engineering Progress (化工 进展),22(1),8-12. (in Chinese with English abstract)

Wolfgang F, Wilfried L (1998). Recent developments and perspectives of industrial rapeseed breeding. Lipid-Fett, 100, 219 226.

World Meteorological Day: Insight to renewable energy resources (世界气象日: 我们的目光投向可再生能源) (2005). http://www. greenpeace. org/china/zh/press/releases/96050. Cited 23 Mar.

Yi H(伊红) (2003). Biodiesel in Germany. Journal of Cereals and Oils (粮食与油脂), (1),32. (in Chinese)

Zhang WY (章文译) (2002). Biodiesel utilized in the occident.
Shiyou Shangbao (石油商报), April 4(in Chinese)

Zhang XM(张小明) (2004). What could be fueled to cars in future? China Automarket (中国汽车市场), (12), 58 - 59. (in Chinese)

Zhao ZR(赵卓然) (2003). New technics developed in Canada to produce biodiesel. Shengli Refining Science and Technology (胜 炼科技), 25(1), 16. (in Chinese)

Zhu JL(朱建良), Zhang GJ (张冠杰) (2004). Current situation and development trend of the research and production of biodiesel. Chemical Industry Time (化工时刊), 18(1),23-27. (in Chinese with English abstract)

Zaffroni E, Kaufman KR, Pratt GL (1987). Vegetable oils as substitutes for diesel oil in Brazil. Applied Engineering in Agriculture, 3, $265-269$. 